Pesq. Vet. Bras. 30(8):665-669, agosto 2010

\title{
Monitoração e avaliação clínica da eficácia da transfusão de sangue total e concentrado de hemácias em cães ${ }^{1}$
}

\author{
Marcel K. Morikawa ${ }^{2}$, Mariana M. Bochio ${ }^{3}$, Vanessa A. Pincelli ${ }^{4}$, Roberta L. \\ Freire $^{5}$ e Patrícia M. Pereira $6^{*}$
}

\begin{abstract}
Morikawa M.K., Bochio M.M., Pincelli V.A., Freire R.L. \& Pereira P.M. 2010. [Monitoring and evaluation of the efficacy of whole blood and packed red cells transfusion in dogs.] Monitoração e avaliação clínica da eficácia da transfusão de sangue total e concentrado de hemácias em cães. Pesquisa Veterinária Brasileira 30(8):665-669. Departamento de Clínicas Veterinárias, Universidade Estadual de Londrina, Campus Universitário, Cx. Postal 6001, Londrina, PR 86051-990, Brazil. E-mail: pmendes@uel.br

Transfusion therapy has a great potential to save lives in small animals practice, and it has been used mainly in the emergency treatment for anemic animals, although this procedure is related to certain risks. A manner to minimize these risks is through keeping close monitoring during the time of transfusion, which furthermore allows an evaluation of recovering of the patient. The aim of the present research was to evaluate the efficacy of whole blood and packed red cells transfusions in anemic dogs. Seventy-seven transfusions in dogs have been done, 52 of whole blood and 25 of packed red cells, measuring (every 15-30 minutes) temperature, cardiac and breathing frequency, mucous tissue color and capillaries filling time throughout the procedure. The outcome shows that both compounds, may cause improvements to every assessed parameters 45 minutes from the start of the transfusion mainly to cardiac frequency, mucous tissue color and capillaries filling time $(p<0.05)$. The success of a transfusion ensures apparent clinical improvement from two hours of the onset of the procedure.
\end{abstract}

INDEX TERMS: Transfusion, whole blood, packed red cells, monitoring, dogs.

RESUMO.- A terapia transfusional tem grande potencial de salvar vidas na clínica de pequenos animais, e é usada principalmente na terapêutica de emergência em animais anêmicos, entretanto este procedimento está associado a vários riscos. Uma das formas de minimizá-los é pela

\footnotetext{
${ }^{1}$ Recebido em 8 de outubro de 2009.

Aceito para publicação em 26 de abril de 2010.

2 Graduado em Medicina Veterinária pela Universidade Estadual de Londrina (UEL), Londrina, PR. Endereço particular: Rua Ipiranga 1030, Jardim Santista, Mogi das Cruzes, SP 08730-000, Brasil.

${ }^{3}$ Graduada em Medicina Veterinária pela Universidade Estadual de Londrina (UEL), Londrina, PR. Endereço particular: Rua Prefeito Hugo Cabral 885, Apto 24, Centro, Londrina, PR 86020-915, Brasil.

${ }^{4}$ Graduada em Medicina Veterinária pela UEL, Londrina, PR. Endereço particular: Rua Prof. João Cândido 668, Apto B3, Centro, Londrina, PR 86010-000.

${ }^{5}$ Departamento de Medicina Veterinária Preventiva (DMVP), CCAUEL, Rodovia Celso Garcia Cid, PR 445 Km 380, Campus Universitário, Cx. Postal 6001, Londrina, PR 86051990.

${ }^{6}$ Departamento de Clínicas Veterinárias, CCA-UEL, Londrina, PR. *Autor para correspondência:pmendes@uel.br
}

monitoração constante durante a transfusão sanguínea, permitindo assim avaliar a melhora clínica do paciente. $\mathrm{O}$ objetivo deste trabalho foi avaliar a eficácia da transfusão de sangue total e de concentrado de hemácias em cães anêmicos, por meio da monitoração dos parâmetros vitais. Foram avaliadas 77 transfusões em cães, sendo 52 de sangue total armazenado e 25 de concentrado de hemácias. Durante todo o procedimento aferiu-se (a cada 1530 minutos) a temperatura, frequência cardíaca, frequência respiratória e a coloração de mucosas dos pacientes, além do tempo de preenchimento capilar. Os resultados obtidos mostraram que ambos os componentes promoveram melhora dos parâmetros avaliados a partir de 45 minutos do início da transfusão sanguínea, principalmente da frequência cardíaca, coloração de mucosas e tempo de preenchimento capilar $(p<0,05)$. A transfusão bem sucedida proporcionou melhora clínica aparente a partir de duas horas do início do procedimento.

TERMOS DE INDEXAÇÃO: Transfusão sanguínea, sangue total, concentrado de hemácias, monitoração, cães. 


\section{INTRODUÇÃO}

A maior incidência de transfusão sanguínea é em pacientes com anemia decorrente de perda de sangue, hemólise ou não produção de eritrócitos pela medula óssea (Knottenbelt \& Mackin 1998, Pereira 2008). As alterações clínicas geralmente estão relacionadas à menor oxigenação dos tecidos e aos mecanismos compensatórios, tais como aumento no débito cardíaco, diminuição da viscosidade do sangue, diminuição da afinidade do oxigênio à hemoglobina e vasoconstrição periférica (Allen \& Allen 1982, Jutkowitz 2005).

Portanto, as principais alterações clínicas são palidez de membranas mucosas, apatia, menor tolerância ao exercício, aumento da frequência respiratória, aumento da frequência cardíaca e sopros cardíacos (Thrall 2007). A indicação da transfusão de sangue deve ser baseada principalmente no histórico e severidade dos sinais clínicos, além dos parâmetros laboratoriais do animal (Pereira \& Ramalho 2001).

É importante monitorar o paciente que recebe transfusão sanguínea para detectar precocemente a ocorrência de reações transfusionais e avaliar sua melhora clínica (Kristensen \& Feldman 1997). A avaliação pré transfusional do paciente pode ser realizada pelo volume globular, proteínas totais, coloração de mucosas, frequências cardíaca e respiratória, temperatura e coloração da urina (Chiaramonte 2004). Além disso, recomenda-se a obtenção do histórico de transfusões prévias, de possíveis reações transfusionais ocorridas e suspeita de doenças hematológicas imunomediadas, devido a sua influência na sobrevivência das células transfundidas (Kristensen \& Feldman 1995).

Durante a transfusão, é importante a avaliação a cada 15 minutos, das frequências cardíaca, respiratória e temperatura na primeira hora e na ausência de reações adversas, a cada hora do restante da transfusão. Caso haja alguma reação, deve-se interromper o procedimento imediatamente, administrar soluções cristalóides intravenosas e se possível, avaliar o débito urinário e a pressão sanguínea. Durante o período pós transfusional, deve-se avaliar a frequência respiratória, coloração de mucosas e o tempo de preenchimento capilar, notando quaisquer alterações no paciente (Kristensen \& Feldman 1995).

Este trabalho objetivou avaliar a eficácia da transfusão de sangue total (ST) e concentrado de hemácias $(\mathrm{CH})$ em cães anêmicos por meio da monitoração de parâmetros vitais e melhora clínica do paciente, durante o período transfusional.

\section{MATERIAL E MÉTODOS}

Foram avaliadas 77 transfusões sanguíneas em cães, realizadas no Hospital Veterinário da Universidade Estadual de Londrina (UEL), Paraná, sendo 52 de ST e 25 de $\mathrm{CH}$, separado por meio de centrifugação a $2500 \mathrm{rpm}$ durante 15 minutos. Foram aferidos os seguintes parâmetros dos pacientes: temperatura $\left(\mathrm{T}^{\circ} \mathrm{C}\right)$, frequência cardíaca $(\mathrm{FC})$, frequência respiratória (FR), coloração das mucosas e tempo de preenchimento capilar (TPC), antes do início da transfusão, a cada 15 minutos durante a primeira hora e a cada 30 minutos até o término do procedimento. Todas as avaliações foram anotadas de forma individualizada, em ficha própria (Fig.1). O

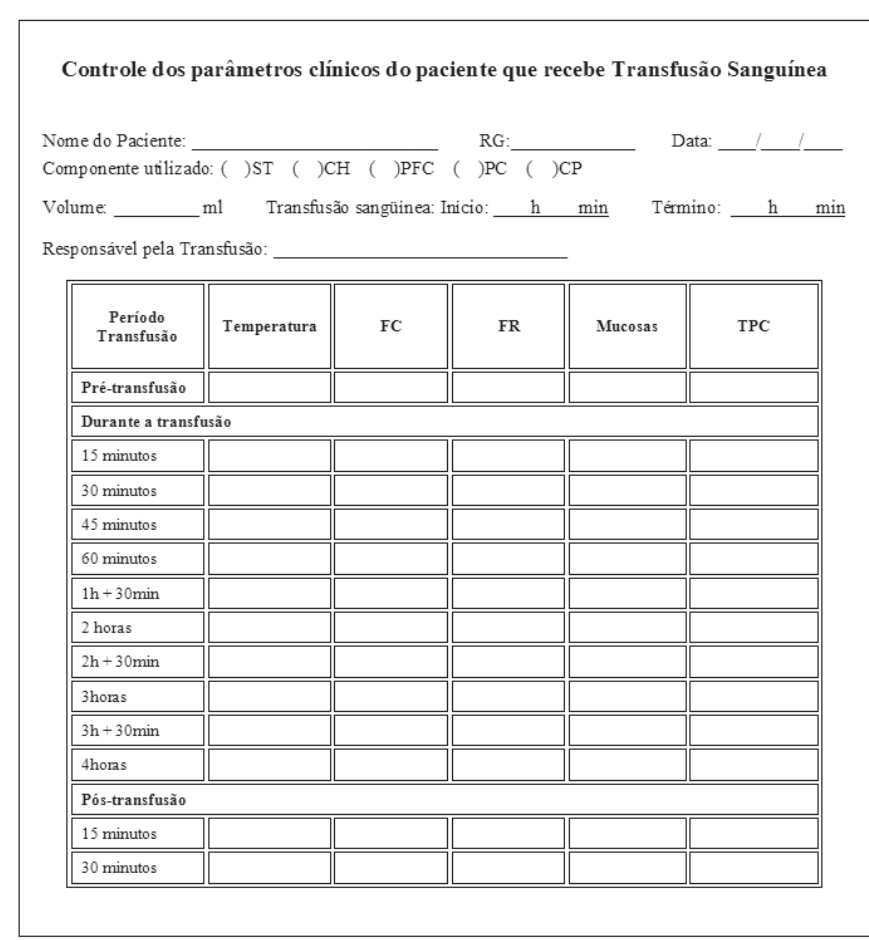

Fig.1. Ficha de controle dos parâmetros clínicos do paciente que recebe Transfusão Sanguínea utilizada no Hospital Veterinário da Universidade Estadual de Londrina (UEL).

tempo máximo de transfusão foi de quatro horas, minimizando desta forma a contaminação bacteriana. Para avaliação da $\mathrm{FC}, \mathrm{FR}$ e $\mathrm{T}^{\circ} \mathrm{C}$ foi utilizado o teste ANOVA de fator único. Para coloração de mucosas e TPC utilizou-se o teste de quiquadrado para a comparação de proporções. Adotou-se o nível de significância de 5\%.

\section{RESULTADOS E DISCUSSÃO}

A anemia causa aumento da FR, FC e aumento do débito cardíaco, porém, estes mecanismos compensatórios nem sempre são suficientes para prevenir a hipóxia tecidual (Toy et al. 2000, Weiskopf et al. 2003). Na Medicina Veterinária há poucos estudos sobre monitoração e tempo na melhora dos parâmetros vitais em cães anêmicos durante a transfusão sanguínea (Pereira 2007).

Do início ao fim da transfusão, o aumento médio da temperatura foi de $0,5^{\circ} \mathrm{C}\left(37,5^{\circ}\right.$ para $\left.38,0^{\circ} \mathrm{C}\right)$ com ST, e de $0,7^{\circ} \mathrm{C}\left(37,3^{\circ}\right.$ para $\left.38,0^{\circ} \mathrm{C}\right) \mathrm{com} \mathrm{CH}$ (Fig.2). A FR diminuiu cinco movimentos respiratórios por minuto $(\mathrm{mrm})$ (33 para $28 \mathrm{mrm}$ ) com ST, e $18 \mathrm{mrm}$ (47 para $29 \mathrm{mrm}$ ) com CH (Fig.3). A FC apresentou diminuição de 35 batimentos por minuto (bpm) (149 para 114bpm) e de 40 bpm (146 a 106 bpm), com ST e CH, respectivamente (Fig.4).

A FC apresentou melhora significativa aos 90 minutos, em relação ao período pré transfusional, tanto com ST $(p=0,0001)$ quanto com $\mathrm{CH}(p=0,007)$, corroborando com os dados de um estudo em que foi observada relação linear entre a anemia e o aumento da FC (Weiskopf et al. 2003). Uma das causas da diminuição da FC é o deslocamento de líquido do espaço extravascular para o espaço 


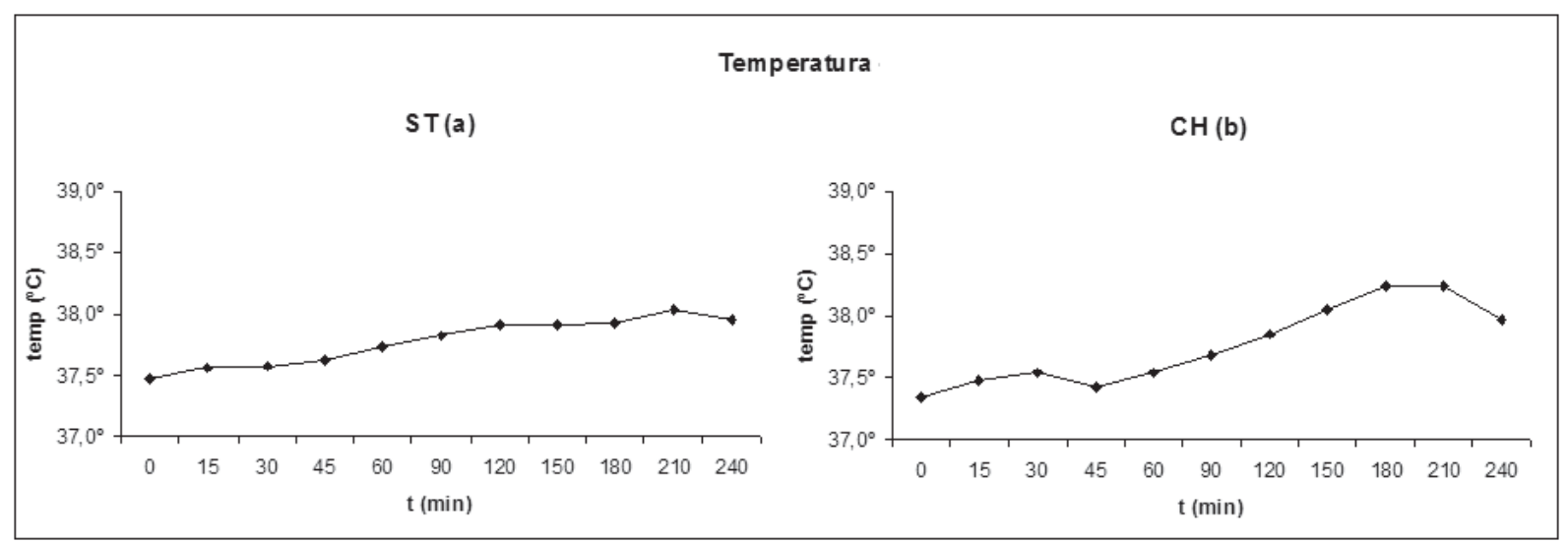

Fig.2. A evolução da temperatura (média) dos animais avaliados, de acordo com o tempo de transfusão sanguínea com ST(a) e CH(b). Londrina, 2009.

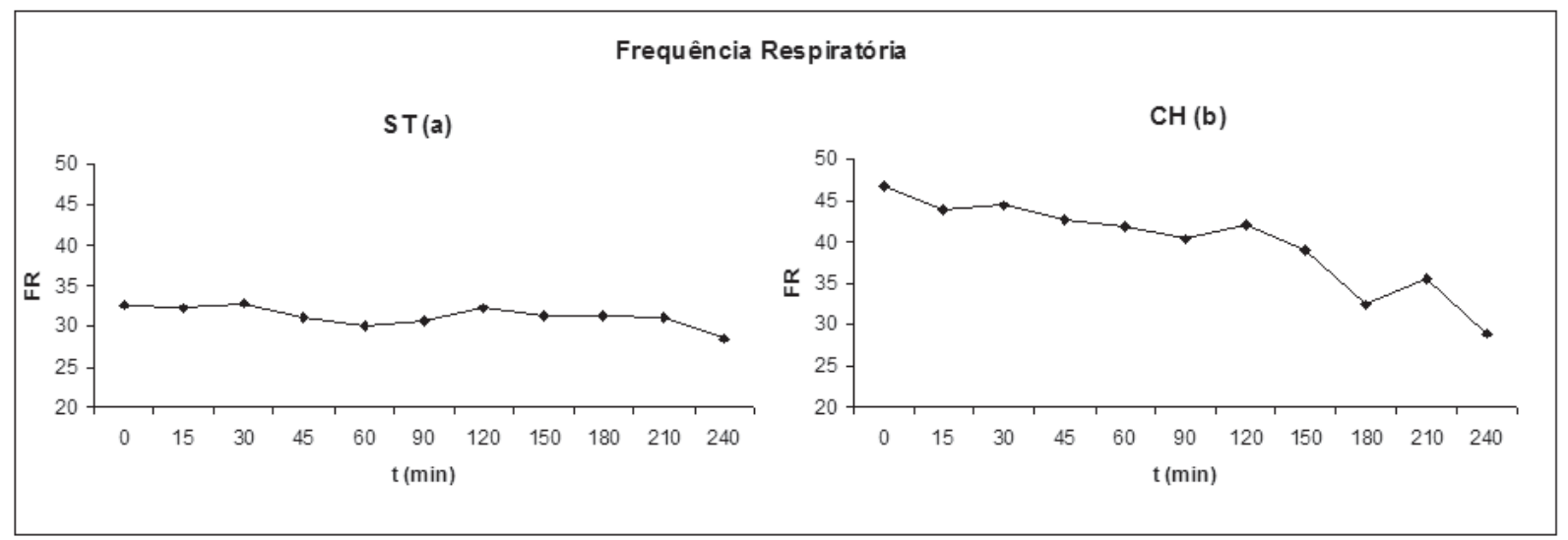

Fig.3. A evolução da frequência respiratória (média) dos animais avaliados, de acordo com o tempo de transfusão sanguínea com ST(a) e $\mathrm{CH}(\mathrm{b})$. Londrina, 2009.

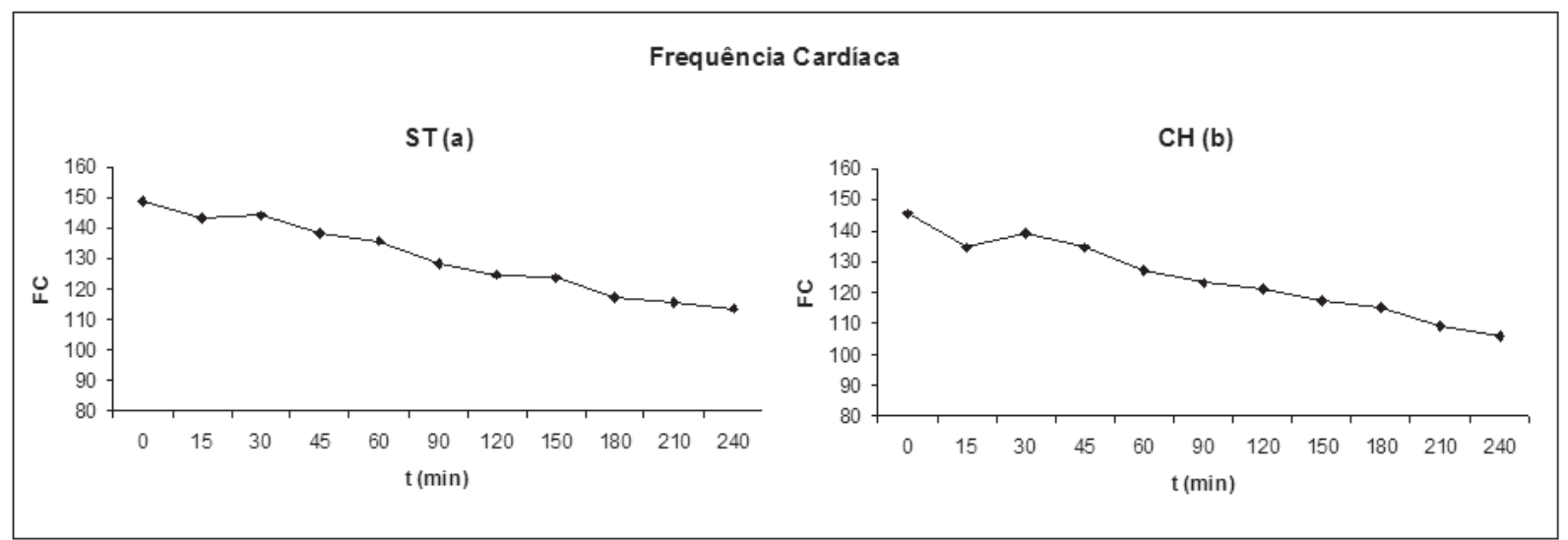

Fig.4. A evolução da frequência cardíaca (média) dos animais avaliados, de acordo com o tempo de transfusão sanguínea com ST(a) e $\mathrm{CH}(\mathrm{b})$. Londrina, 2009.

intravascular, estimulado pela infusão de grandes quantidades de sangue (Raiser 2005). Porém, neste estudo, embora não se possa descartar esta afirmação, a melhora significativa da FC ocorreu aos 90 minutos, e neste período o volume infundido era pequeno, visto que nos primeiros 30 minutos a velocidade de infusão do sangue foi de aproximadamente 0,25 a $0,5 \mathrm{~mL} / \mathrm{kg} / 30 \mathrm{~min}$.
Conforme se observa na Figura 5, no início da transfusão com ST, 92,31\% dos cães apresentaram mucosas de coloração pálida e 7,69\% rósea clara. A partir dos 45 minutos observou-se melhora significativa deste quadro, sendo que $71,15 \%$ apresentaram mucosas pálidas e $23,08 \%$ róseas claras $(\mathrm{p}=0,009)$. Aos 90 minutos do início da transfusão, $21,57 \%$ apresentavam mucosas róseas $(p=0,003)$. 


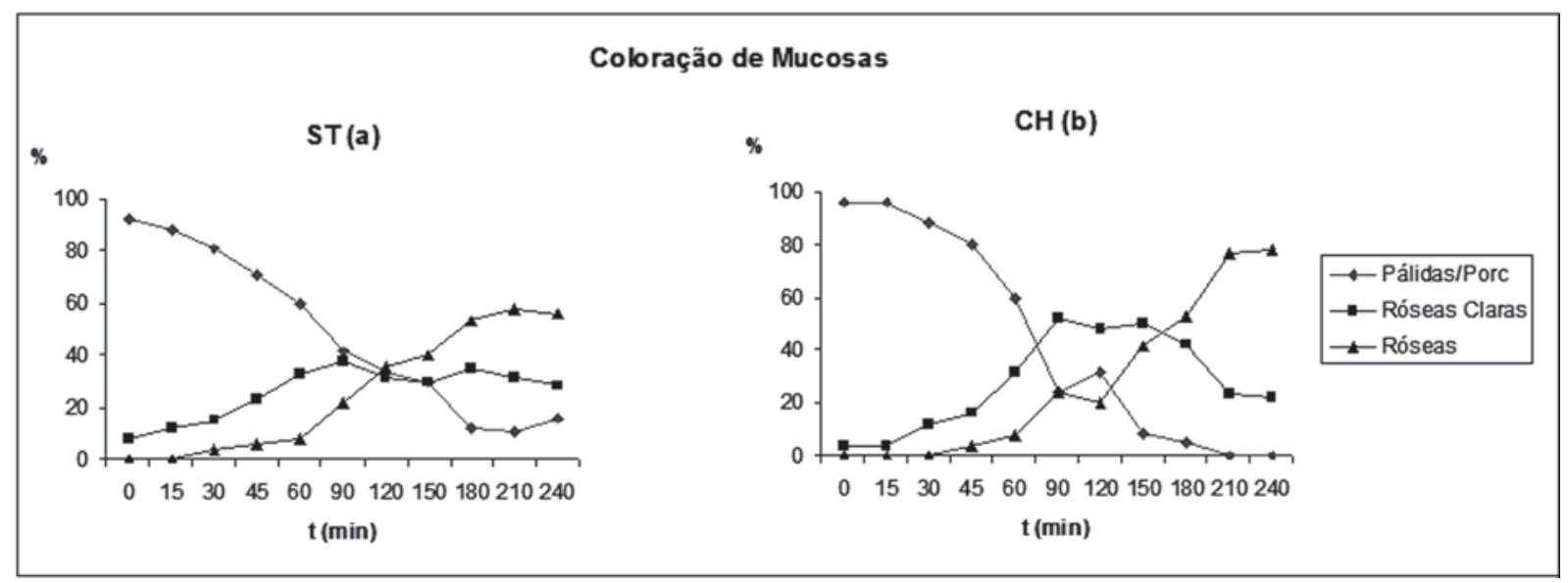

Fig.5. A evolução de acordo com o tempo de transfusão sanguínea com ST(a) e $\mathrm{CH}(\mathrm{b})$, para coloração de mucosas (média): \% de animais para cada classificação. Londrina, 2009.

Ao final da transfusão, 16,0\% apresentaram mucosas pálidas, $28,0 \%$ mucosas róseas claras e $56,0 \%$ dos cães apresentaram mucosas róseas $(p<0,0001)$. No início das transfusões com $\mathrm{CH}, 96,0 \%$ dos animais apresentaram

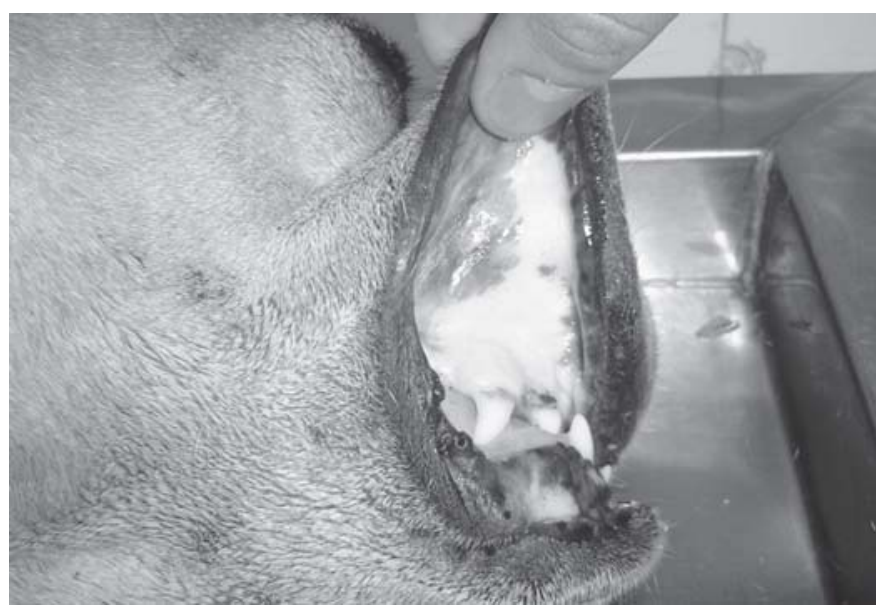

Fig.6. Coloração de mucosas (pálidas), início da transfusão. Fonte: Patrícia Mendes Pereira.

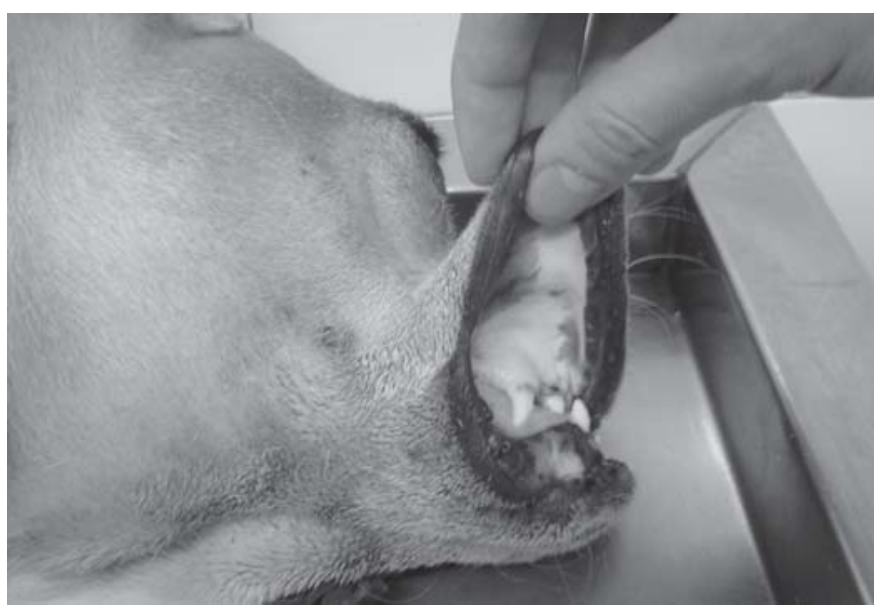

Fig.7. Coloração de mucosas (róseas claras), 60 minutos após início da transfusão. Fonte: Patrícia Mendes Pereira.

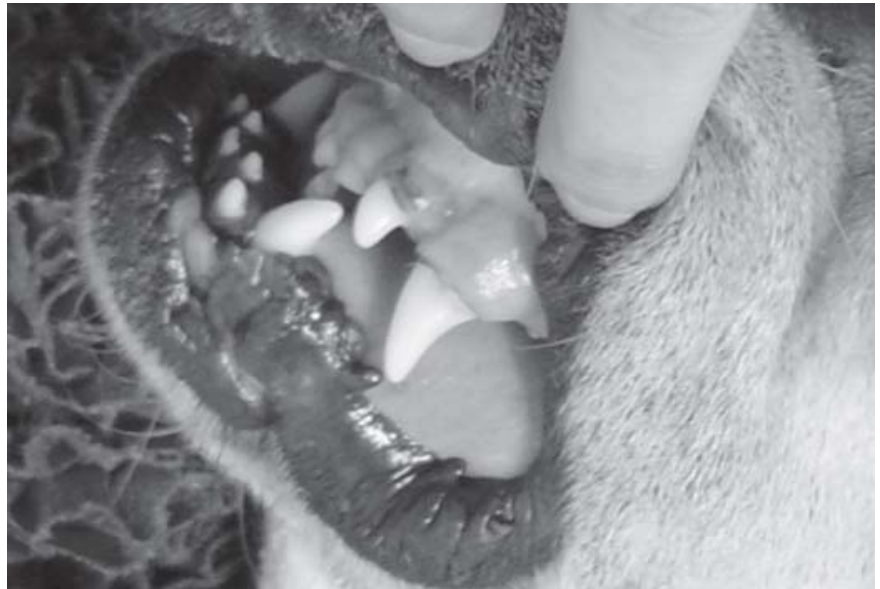

Fig.8. Coloração de mucosas (róseas), 120 minutos após início da transfusão. Fonte: Patrícia Mendes Pereira.

mucosas de coloração pálida. A partir dos 60 minutos observou-se alteração significativa, sendo que $60 \%$ apresentaram mucosas pálidas e $32,0 \%$ róseas claras $(p=0,022)$. Aos 90 minutos, $24,0 \%$ apresentaram mucosas róseas $(p=0,05)$. Ao final da transfusão, $22,22 \%$ apresentaram mucosas róseas claras e $77,78 \%$ mucosas róseas e nenhum animal apresentou mucosa pálida $(p<0,0001)$. É importante salientar que o tempo de transfusão usado neste estudo variou de três a quatro horas. Desta forma, podemos afirmar que a melhora clínica na maioria dos cães ocorreu antes da metade do tempo total da transfusão, demonstrando a eficácia deste procedimento (Fig.6-8).

O TPC teve diminuição significativa a partir de $90 \mathrm{mi}-$ nutos do início da transfusão, em 50,98\% ( $p=0,019)$ e $60 \%$ $(p=0,02)$ dos animais transfundidos por ST e CH, respectivamente (Fig.9). Esta melhora pode ser decorrente da infusão de fluido coloidal e deslocamento de líquido do espaço extravascular para o espaço intravascular (Raiser 2005). Entretanto, em muitos pacientes em questão, a avaliação do TPC foi prejudicada em decorrência da palidez da mucosa.

Outro parâmetro verificado, porém, não avaliado esta- 


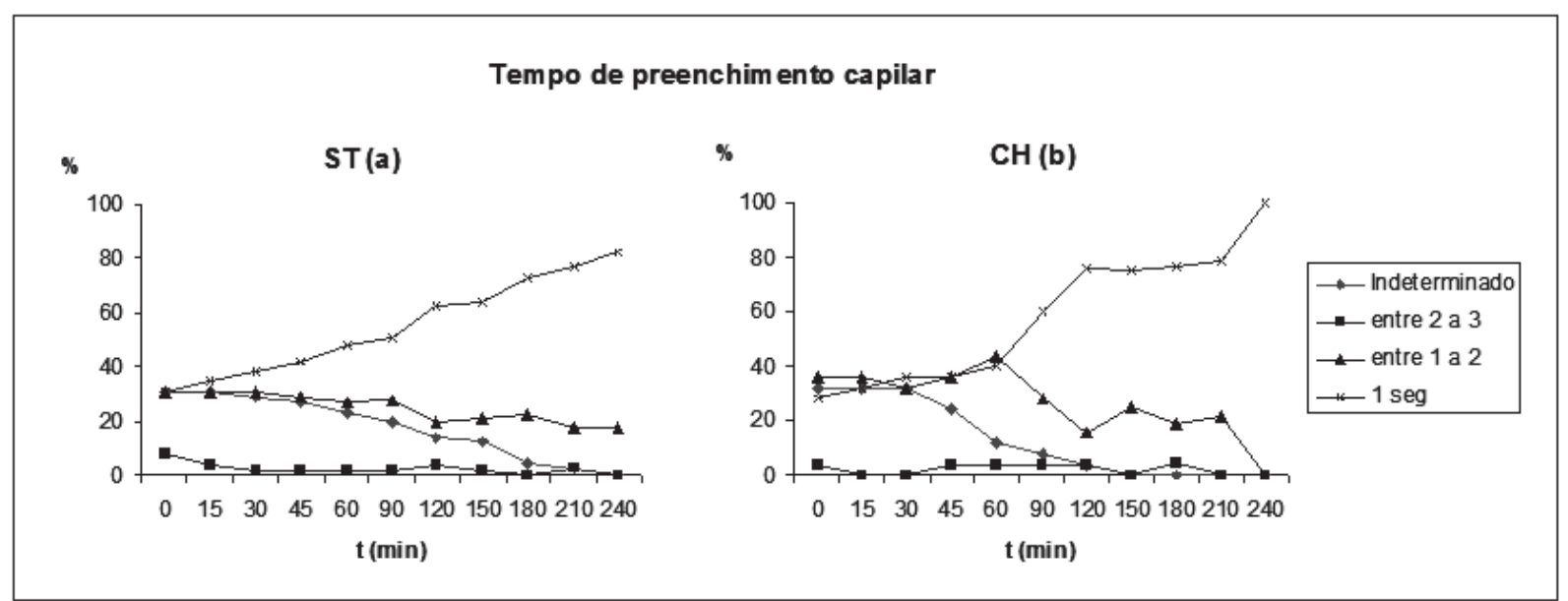

Fig.9. A evolução de acordo com o tempo de transfusão sanguínea com ST(a) e $\mathrm{CH}(\mathrm{b})$, para tempo de preenchimento capilar (média): \% de animais para cada classificação. Londrina, 2009.

tisticamente, foi a diminuição da apatia e prostração do paciente, que começa a ser observada após uma a duas horas do início da transfusão de sangue.

\section{CONCLUSÕES}

A transfusão sanguínea promove melhora clínica para pacientes anêmicos enquanto se busca o diagnóstico ou até que o tratamento se torne efetivo.

Por meio da monitoração constante, é possível avaliar a eficácia ou não da transfusão sanguínea.

Portanto, a verificação dos parâmetros vitais (FC, FR, $\mathrm{T}^{\circ} \mathrm{C}$, TPC e coloração de mucosas) revela que a transfusão bem sucedida proporciona melhora clínica aparente a partir de duas horas do início do procedimento.

Caso isso não ocorra, deve-se ficar atento à possibilidade de ocorrência de reações adversas.

\section{REFERÊNCIAS}

Allen J.B. \& Allen F.B. 1982. The Minimum acceptable level of hemoglobin. Int. Anesthesiol. Clinics 20:1-22. (Apud Hohenhaus 1992)

Chiaramonte D. 2004. Blood-Component Therapy: Selection, administration and monitoring. Clin. Tech. Small Anim. Pract. 9:6367.

Hohenhaus A.E. 1992. Canine blood transfusions, p.612-624. In: Ibid. (Ed.), Transfusion Medicine, Problems in Veterinary Medicine. $4^{\text {th }}$ ed. Lippincott Company, Philadelphia.

Jutkowitz L.A. 2005. Transfusion Triggers for the Emergent Patient.
International Veterinary Emergency and Critical Care Symposium, p.221-226.

Knottenbelt C. \& Mackin A. 1998. Blood transfusions in the dog and cat. 2. Indications and safe administration. In Practice 20:191-199.

Kristensen A.T. \& Feldman B.F. 1997. Bancos de sangue e medicina transfusional, p.497-516. In: Ettinger S.J. \& Feldman E.C. (Eds), Tratado de Medicina Interna Veterinária. $4^{\mathrm{a}}$ ed. Manole, São Paulo.

Kristensen A.T. \& Feldman B.F. 1995. General Principles of small animal blood component administration. Vet. Clin. North Am., Small Anim. Pract. 25:1277-1290.

Pereira P.M. 2008. Transfusão de sangue e seus derivados, p.579589. In: Andrade S.F. (Ed.), Manual de Terapêutica Veterinária. $3^{\underline{a}}$ ed. Roca, São Paulo.

Pereira P.M. 2007. Transfusões em cães e gatos, p.18-26. In: Lopes S.T., Biondo A.W. \& Santos A.P. (Eds), Manual de Patologia Clínica Veterinária. $3^{\mathrm{a}}$ ed. UFSM, Santa Maria.

Pereira P.M. \& Ramalho F.S. 2001. Transfusão sanguínea. Revta Clín.Vet. 6:34-40.

Raiser A.G. 2005. Choque, p.71-104. In: Rabelo R.C. \& Crowe D.T. Jr (Eds), Fundamentos de Terapia Intensiva Veterinária em Pequenos Animais. L.F. Livros, Rio de Janeiro.

Thrall M.A. 2007. Classificação e diagnóstico de anemia, p.78-83. In: Ibid. (Ed.), Hematologia e Bioquímica Clínica Veterinária. Roca, São Paulo.

Toy P., Feiner J., Viele M.K., Watson J., Yeap H. \& Weiskopf R.B. 2000. Fatigue during acute isovolemic anemia in healthy, resting humans. Transfusion 40:457-460.

Weiskopf R.B., Feiner J., Hopf H., Viele M.K., Watson J.J., Lieberman J., Kelley S. \& Toy P. 2003. Heart rate increases linearly in response to acute isovolemic anemia. Transfusion 43:235-240. 